\title{
A Novel Biofortification of Selenium Nanoparticles (SeNPs) in Drinking Water and Examination of its Hepatotoxicity in Wister Rats
}

\author{
Subburaman Chandramohan, Krishnan Sundar, Azhaguchamy Muthukumaran
}

\begin{abstract}
Biofortification is the delivering micronutrients to populations that may have limited access to diverse diets. Selenium is one of the vital micronutrients that is indispensable to human health and protects the cells by preventing the formation of free radicals. Nanoform of Selenium could be used for biofortification process. In the present work selenium nanoparticles (SeNPs) were synthesized by lemon extract, which acted both as reducing and capping agent. The synthesized SeNPs were characterized by Fourier Transformer InfraRed Spectroscopy (FTIR) and Scanning Electron Microscopy (SEM) coupled with Energy Dispersive $X$ ray analysis (EDX). FTIR studies revealed the presence of functional groups responsible for nano particle reduction in the nanoparticle formation. The elemental analysis of SeNPs as well as mapping were also carried out which revealed the presence of selenium in the nano suspension. The average size of the synthesized SeNPs was found to be in the range of 200-250 $\mathrm{nm}$ with spherical in shape. Furthermore, the prepared SeNPs was fortified in drinking water and given to Wister rats for 21day period, and their effects studied in livers of the Wister rats. The liver histopathology analysis showed that, SeNPs possesses very minimal or no toxicity, when compared to ionic form of selenium given in rats.

Keywords: Selenium Nanoparticles (SeNPs), Sodium selenite, Biofortification, Wister rat, Histopathology.
\end{abstract}

\section{INTRODUCTION}

Selenium is one of the biologically important trace elements which is significant to the human health [1]. Because of its potential as a nano medicine higher preference is given to produce selenium at nano sizes. Se-proteins is been involved in antioxidant activities and redox regulation, especially glutathione peroxidases and thioredoxin reductases [2]. In the past two decades, there are many studies available to suggest that insufficient Se levels, and particularly Se-proteins, are directly related with several diseases such as cancer, diabetes, cardiovascular and immune system malfunctions in humans [3]. There were many studies which suggest the beneficial effect of selenium from general health protection and its deficiency levels. The absence or less

Revised Manuscript Received on December 05, 2019

Subburaman Chandramohan, Department of Biotechnology, Kalasalingam Academy of Research and Higher Education, Krishnankoil, Tamil Nadu 626126, India.

Krishnan Sundar, Department of Biotechnology, Kalasalingam Academy of Research and Higher Education, Krishnankoil, Tamil Nadu 626126, India.

Azhaguchamy Muthukumaran*, Department of Biotechnology, Kalasalingam Academy of Research and Higher Education, Krishnankoil, Tamil Nadu 626126, India. intake levels of selenium in the diet are associated with various cancers and even absence of selenium intake is related with the development of AIDS in HIV patients [4]. The abundant sources of selenium are available in sea-foods and also in organ meats. Other sources of selenium include muscle meats, cereals, grains and leafy vegetables [5]. The quantity of selenium present in a plant depends on the quantity of selenium in the soil and several other factors such as soil $\mathrm{Ph}$, amount of organic matter. Water is an indispensable constituent for the survival of humans and genesis of organisms. Fortification is nothing but, adding essential nutrients to a food, whether or not it is normally contained in the food for correcting demonstrated deficiency of one or more nutrients [6]. Evolution of fortified water started in 7000 years ago where which, water is mixed with grape wine. Microorganisms present in the water were eliminated by this technique [7]. The National Institutes of Health recommends that people at the age of 14 to get atleast 55 micrograms of selenium per day [8]. For women who are pregnant or lactating, this increases to 70 micrograms [9]. Selenium deficiency is associated with the progression of keshan disease, a condition of cardiomyopathy [10] Selenium deficiency is also related to male infertility and also play a role in keshin-beck disease- a type of osteoarthritis [11]. Nanotechnology is the ability to measure, manipulate and manufacture things on an atomic or molecular scale, usually between 1 and 100 nanometers. Nanotechnology also plays a central role in the manufacture of mechanical, optical and electronic devices, biotechnology, microbiology, bio remediation, medicine, numerous engineering fields and material science [12]. When converting ionic form of selenium into its nano form its efficiency can be increased significantly [13]. The use of plant extract for the synthesis of metal nanoparticles is the trend now because of its efficiency as a reducing agent and minimal toxicity compared to chemical compounds used as reducing agents in nanoparticle synthesis. Wister rats are one of the well characterized and studied strain, which usually shows stable control data in most of the experiments. Other advantages of using Wister rats are their higher survival rates, lower body weight compared to other experimental rat strains and they are relatively easy to breed in laboratory conditions [14]. In the present work, we synthesized 
SeNPs by aqueous lemon extract which acts both as reducing and capping agent. The synthesized SeNPs were characterized by SEM, EDX and FTIR analysis. The synthesized SeNPs were fortified in purified drinking water and the hepatotoxicity of the SeNPs fortified drinking water was analyzed in a Wister rat model.

\section{MATERIALS AND METHODS}

\section{A. Synthesis of selenium nanoparticles (SeNPs)}

For preparation of SeNPs of Stock concentration of $2 \mathrm{Mm}$, $0.1 \mathrm{~g}$ of sodium selenite was dissolved in $25 \mathrm{ml}$ of double distilled water. The sodium selenite stock solution was mixed with $25 \mathrm{ml}$ of lemon extract and heat sterilized in an autoclave for $15 \mathrm{~min}$. The obtained reaction mixture was centrifuged for $10000 \mathrm{rpm}$ at $4^{\circ} \mathrm{C}$. The washing step was repeated atleast three times to get a clear nanosuspension which was stored for further studies.

\section{B. Characterization of synthesized SeNPs}

The prepared SeNPs were characterized by FTIR, XRD, SEM and EDX analysis.

3.1 FTIR analysis

The synthesized SeNPs were analyzed for the presence of functional groups using Fourier Transform- Infrared Spectrophotometer (IR Tracer-100 Shimadzu, Japan) ranging from $400 \mathrm{~cm}^{-1}$ to $4000 \mathrm{~cm}^{-1}$.

\section{SEM analysis}

The morphology and size of the SeNPs were measured using a Scanning Electron Microscope (Carl Zeiss Evo 18, Germany). This selected areas within the SEM sections were subjected to elemental composition analysis using an Energy Dispersive X-ray spectroscopy (Quantax 200 with X-Flash, Bruker, USA) system coupled with SEM.

\section{Histopathology}

After the experimental period (21 days) the rats were anesthetized and liver was surgically removed from the Control and the treatment groups. The harvested liver samples were stored immediately in $10 \%$ formalin subjected to histopathology analysis. The histopathological sections were prepared according to the modified protocol of Wen et al [15]. Finally, fixed tissues were observed under light microscope (Primostar, Carl Zeiss, Germany). The experiments in Wister rats were strictly performed according to Institutional Animal Ethical Committee (AKCP/IAEC/74/2018-19).

\section{RESULTS AND DISCUSSION}

\section{A. Synthesis of SeNPs}

In this study, Sodium selenite reacted with lemon extract and yielded SeNPs. Here lemon extract acts as a reducing agent as well as capping agent for the formation of SeNPs (Figure 1 shows the color change from colorless to reddish brown) via thermal reaction [16]. The synthesis step is followed by purification in which the reaction mixture containing the SeNPs were centrifuged at $1000 \mathrm{rpm}$ for $10 \mathrm{~min}$. The washing step was repeated atleast three times to ensure the purity of SeNPs.

\section{B. Characterization of synthesized SeNPs}

The FTIR Spectra showed the presence of several bands for the corresponding functional groups in aqueous solution of SeNPs. IR peaks for $-\mathrm{OH}$ stretching of water was observed at around $3321 \mathrm{~cm}^{-1}$. Nitrile carbenes were present and conformed by stretching were observed at around 2034.90 $\mathrm{cm}^{-1}, 2139.06 \mathrm{~cm}^{-1}, 2158.35 \mathrm{~cm}^{-1}, 2189.21 \mathrm{~cm}^{-1}$. The other peaks such as $\mathrm{C}=\mathrm{O}$ and $\mathrm{C}=\mathrm{C}$ was observed at $2013.68 \mathrm{~cm}^{-1}$ and $1635.64 \mathrm{~cm}^{-1}$ (Fig. 1)

In the present study, the surface morphology of the SeNPs was characterized using SEM analysis. SEM observation of the synthesized SeNPs revealed uniform smooth surfaced spheres. (Fig. 2) showed the Scanning Electron Microscopy of the prepared SeNPs. The EDX analysis spectrum confirmed the presence of SeNPs in the nanosuspension (fig. $3)$.

\section{Histopathology studies in Liver}

In the present study, The Wister rats were given drinking water fortified with sodium selenite and drinking water fortified with SeNPs for 21 days. At the end of the study period, the rats were euthanized and liver samples were collected and histopathology analysis of the liver sections were carried out. In the case of the sodium selenite treated rat, the studied liver sections showed liver parenchyma hepatocytes arranged in trabecular pattern. Close microscopic examination of the section showed significant periportal chronic inflammatory cell infiltration and Congestion [17]. Whereas in the case of SeNPs treated rat's liver section showed liver parenchyma Hepatocytes with no significant pathology.

\section{CONCLUSION}

In the current work, we synthesized Selenium nanoparticles (SeNPs) from natural extracts of lemon which acted as a reducing and capping agent in the reduction of ionic sodium selenite into nano Se. The prepared lemon extract capped SeNPs were fortified with drinking water and the toxicity of the SeNPs fortified drinking water was studied using Wister rat model. Results of the of the toxicity studies strongly implied that the prepared Selenium nanoparticles (SeNPs) is much safer than the existing Se fortified drinking water, which is been selling in the commercial markets right now. 


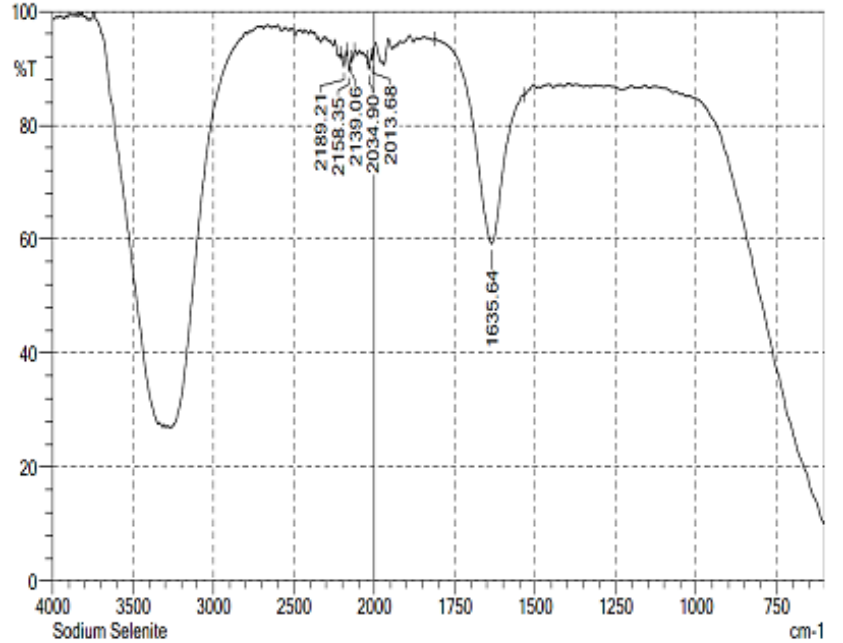

Fig.1. FTIR analysis of SeNPs

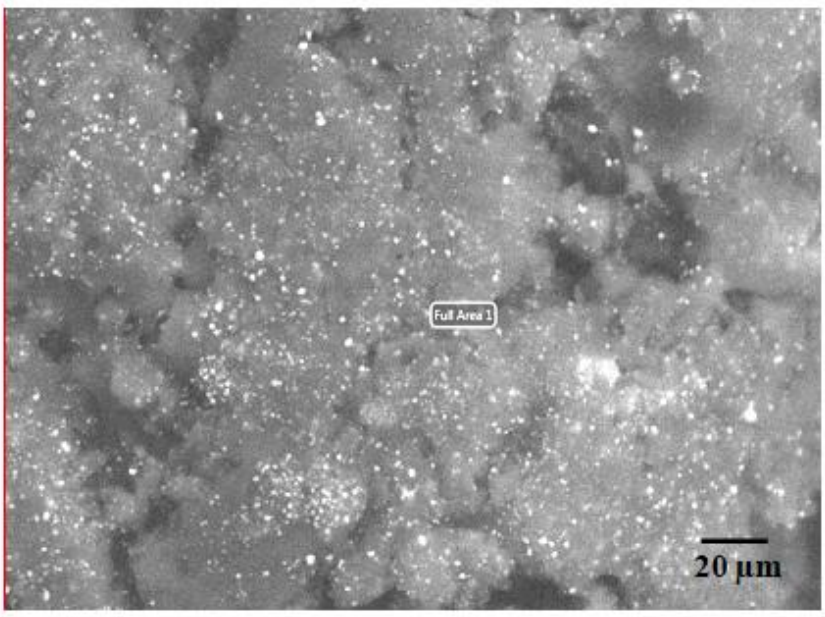

Fig.2. The SEM analysis of SeNPs

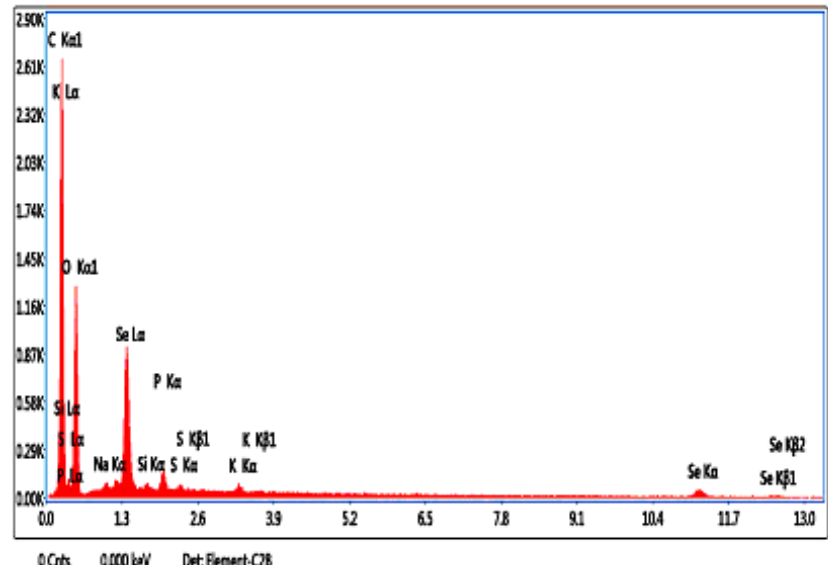

Fig. 3. The EDX mapping, plotted for Se and O peak from SeNPs

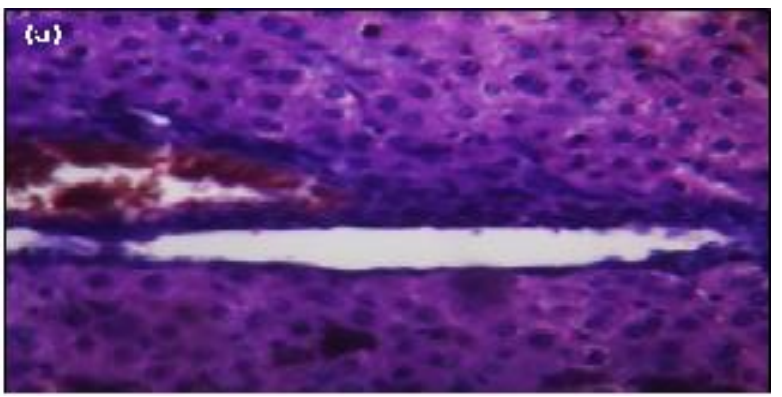

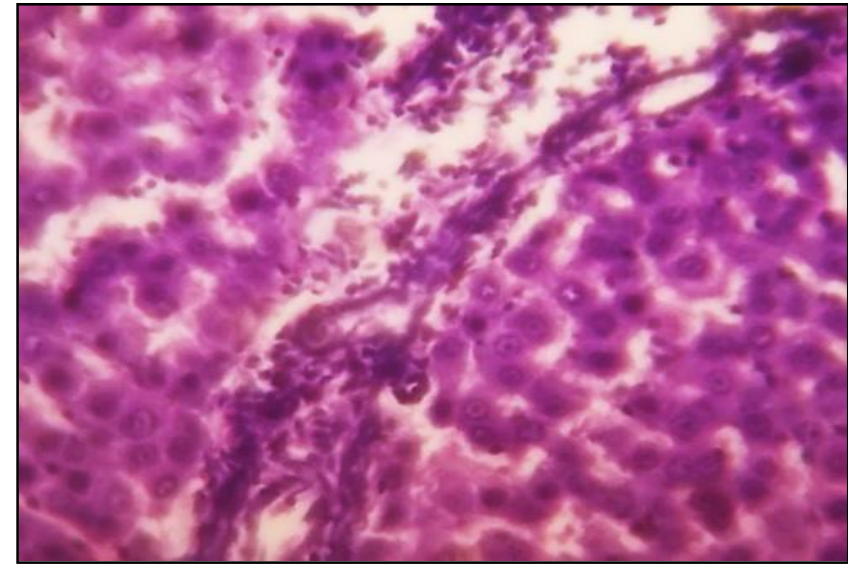

Fig. 4. The histopathology studies of liver sections of Wister rats treated with (a) Sodium Selenite (b) SeNPs.

\section{ACKNOWLEDGMENT}

SC thanks the management of Kalasalingam Academy of Research and Education for University Research Fellowship.

\section{REFERENCES}

1. M.P. Rayman, "The importance of selenium to human health," The lancet vol. 356, pp. 233-241, 2000 .

2. M. Roman, P. Jitaru, and C. Barbante, "Selenium biochemistry and its role for human health," Metallomics, vol. 6, pp. 25-54, 2014.

3. M.P. Rayman, "Selenium and human health," The Lancet, vol. 379, pp. 1256-1268, 2012.

4. C.R. Sudfeld, S. Aboud, R. Kupka, F.M. Mugusi, and WW. Fawzi, "Effect of selenium supplementation on HIV-1 RNA detection in breast milk of Tanzanian women," Nutrition, vol. 30, pp. 1081-1084, 2014

5. J.W. Finley, "Bioavailability of selenium from foods," Nutrition reviews, vol. 64, pp. 146-151, 2006

6. S.M.T. Gharibzahedi and S.M Jafari, "The importance of minerals in human nutrition: Bioavailability, food fortification, processing effects and nanoencapsulation," Trends in Food Science \& Technology, vol. 62 pp.119-132, 2017.

7. R.D. Semba, "The historical evolution of thought regarding multiple micronutrient nutrition," The Journal of nutrition, vol. 142, pp.143S-156S 2011.

8. G.N. Schrauzer, and D.A White, "Selenium in human nutrition: dietary intakes and effects of supplementation," Bioinorganic chemistry, vol. 8 , pp.303-318, 1978

9. A.M. Smithand, M.F Picciano, "Evidence for increased selenium requirement for the rat during pregnancy and lactation," The Journal of nutrition, vol. 116, pp.1068-1079, 1986.

10. J. Loscalzo, "Keshan disease, selenium deficiency, and the selenoproteomes," New England Journal of Medicine, vol. 370 pp.1756-1760, 2014

11. Y. Chen, J.M Zhai, Z.L Wang, X.W Tan, L. Xue and D Geng,. "A comparative research on the treatment effect of Se supplement, Vit C supplement and cereals dryness on Kaschin-Beck disease," Chinese Journal of Control of Endemic Disease, vol. 6, 2003.

12. S. Saif, A. Tahir, and Y. Chen, "Green synthesis of iron nanoparticles and their environmental applications and implications," Nanomaterials, vol. 6 pp.209, 2016

13. W. Majeed, M. Zafar, A. Bhatti, and P. John, "Therapeutic potential of selenium nanoparticles," J. Nanomed. Nanotechnol, vol. 9, 2018

14. K. Weber et al. "Differences in rat models used in routine toxicity studies," International journal of toxicology, vol. 30, pp.162-173, 2011

15. H. Wen et al, "Acute toxicity and genotoxicity of silver nanoparticle in rats," PloS one, vol. 12, pp. e0185554, 2017.

16. S. Chandramohan, K. Sundar and A. Muthukumaran, "Monodispersed spherical shaped selenium nanoparticles (SeNPs) synthesized by Bacillus subtilis and its toxicity evaluation in zebrafish embryos," Materials Research Express, vol. 5, p.025020, 2018.

17. M. Orazizadeh, F. Fakhredini, E. Mansouri, and L. Khorsandi, "Effect of glycyrrhizic acid on titanium dioxide nanoparticles-induced hepatotoxicity in rats," Chemico-biological interactions, vol. 220, pp.214-221, 2014.

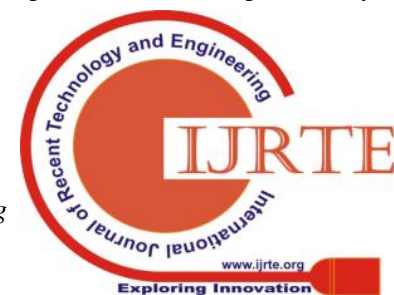




\section{AUTHORS PROFILE}

Mr. S. Chandramohan is currently pursuing Ph.D in Biotechnology at Department of Biotechnology, Kalasalingam Academy of Research and Education. His research interests in Nanobiotechnology, Drug delivery and Safety assessment of nanoparticles.

Dr. K. Sundar is currently working as a Professor in the Department of Biotechnology in Kalasalingam Academy of Research and Education. His research interests in Immunology and Vaccinology.

Dr. A. Muthukumaran is currently working as Associate Professor and Head in the Department of Biotechnology in Kalasalingam Academy of Research and Education. His research interests in Nanobiotechnology, Drug delivery and Safety assessment of nanoparticles 